\title{
The Anterior Cerebellar Vermis: Essential Involvement in Classically Conditioned Bradycardia in the Rabbit
}

\author{
William F. Supple, Jr., and Bruce S. Kapp \\ Department of Psychology, The University of Vermont, Burlington, Vermont 05405
}

\begin{abstract}
The effects of lesions of the cerebellum on the acquisition and retention of aversive Pavlovian conditioned bradycardia were examined in rabbits. Lesions of the anterior cerebellar vermis severely attenuated the acquisition of simple conditioned bradycardia without disrupting baseline heart rate (HR), or unconditioned HR responses. Also, lesions of the vermis performed after the acquisition of conditioned bradycardia eliminated evidence of prior conditioning. Bilateral lesions of the cerebellar hemispheres did not affect conditioned or unconditioned HR responses. These results were interpreted to indicate that anterior vermis lesions specifically disrupted part of an essential conditioned response pathway without interfering with the neural circuits that mediate unconditioned HR responding. These lesion data, coupled with recent electrophysiological evidence of learningrelated changes in neuronal activity within the anterior vermis of the fear-conditioned rabbit, suggest that the anterior cerebellar vermis is critically involved in the acquisition and retention of this rapidly learned autonomic conditioned response.
\end{abstract}

[Key words: cerebellar vermis, conditioned bradycardia, lesions, unconditioned response, cerebellar hemisphere, learning and memory]

The cerebellum contributes to a variety of complex behavioral processes in addition to its well-documented role in skeletal motor regulation and coordination (Dow and Moruzzi, 1958). The midline cerebellum, which includes the vermal cortex and fastigial nuclei, has a functional contribution to a variety of affective and fear-related behaviors (Berman et al., 1974; Berntson and Torello, 1982; Supple et al., 1987). Decreased behavioral reactivity followed lesions of the vermis in cats and monkeys (Peters and Monjan, 1971; Berman et al., 1974), and several fear-related behaviors are attenuated after vermis or fastigial nucleus lesions in rats (Berntson and Torello, 1982; Supple et al., 1987, 1988). Furthermore, stimulation of the midline cerebellum evokes affective behavior that resembles fear-related responses (Asdourian and Frerichs, 1970; Reis et al., 1973; Albert et al., 1985) and also results in many of the autonomic responses that accompany these affective states (Martner, 1975). For example, midline cerebellar stimulation resulted in dra-

\footnotetext{
Received Nov, 12, 1992; revised Jan. 29, 1993; accepted Mar. 11, 1993.

We acknowledge the technical assistance of Lauren Archer. This work was supported by NIMH Grant MH09549 and NIMH FIRST MH47307-01 to W.F.S.

Correspondence should be addressed to William F. Supple, Department of Psychology; John Dewey Hall, The University of Vermont, Burlington, VT $05405-$ 0134.

Copyright (C) 1993 Society for Neuroscience $0270-6474 / 93 / 133705-07 \$ 05.00 / 0$
}

matic changes in heart rate (HR) (Hockman et al., 1970; Hoffer et al., 1972; Nisimaru and Watanabe, 1985), arterial blood pressure (Miura and Reis, 1969; Chida et al., 1986), and respiration rate (Snider, 1972).

This correspondence between the effects of midline cerebellar lesions and stimulation on fear-related behaviors and autonomic responses prompted an examination of the contributions of the vermis to a classically conditioned fear-related autonomic response. Large lesions of the vermis severely attenuated the acquisition of conditioned bradycardia in the restrained rat (Supple and Leaton, 1990a,b). Importantly, the lesions specifically disrupted the conditioned response (CR) without altering unconditioned HR (UCR) responses. Furthermore, within the cerebellum the vermis seems particularly critical as bilateral lesions of the cerebcllar hemisphcres did not disrupt conditioned HR responding (Supple and Leaton, 1990b).

The present experiment examined the contributions of the cerebellum to the acquisition and retention of conditioned bradycardia in the rabbit, a species considered by many as an ideal intact preparation for the study of neural mechanisms of learning and memory, and in particular, cardiovascular conditioning (see Schneiderman et al., 1986; Pascoe et al., 1991). This experiment examined the effects of cerebellar lesions on conditioned bradycardia using a simple, nondiscriminative conditioning paradigm. HR was recorded during several different phases of the conditioning procedure to determine the effects of the lesions on unconditioned and conditioned HR changes. HR was recorded during baseline periods and unreinforced tone conditioned stimulus (CS) presentations to determine if lesions altered resting $H R$, the HR orienting response (HR OR), or its habituation. During acquisition training the unconditioned HR response following the aversive unconditioned stimulus (UCS) was also examined. Measurement of these unconditioned HR responses during the various phases of training should indicate if the lesions have disrupted unconditioned features of the HR response that might complicate the interpretation of any observed conditioning deficit.

Two distinct regions of the cerebellum were lesioned in separate groups. The anterior cerebellar vermis (ACV) was targeted because it was included in the effective lesions in the rat (Supple and Leaton, 1990a,b) and because subsequent electrophysiological data in the rabbit indicated learning-related alterations in neuronal activity within the anterior vermis (Supple and Kapp, 1988). Another group with bilateral cerebellar hemispheric lesions (HEM) was included to serve as a surgical control for any general debilitating effects of cerebellar damage per se, and because previous research (Lavond et al., 1984; Supplc and Leaton, 1990b) has shown similar lesions to be ineffectual on 
autonomic conditioning yet devastating on somatomotor CRs like the nictitating membrane response in the rabbit (McCormick and Thompson, 1984; Yeo et al., 1985).

\section{Materials and Methods}

\section{Subjects}

Experiments were performed on 20 adult New Zealand White rabbits (Oryctolagus cuniculus) weighing between 2.2 and $2.5 \mathrm{~kg}$ and obtained from a local licensed supplier. Animals were maintained on a 12 hr: 12 hr light : dark cycle with food and water available ad libitum. Each rabbit was handled extensively prior to the start of the experiment. Principles for the care and use of laboratory animals as outlined by the U.S. Public Health Service were strictly followed.

\section{Surgery}

At least 2 weeks before the initiation of behavioral training, the animals were surgically prepared. After application of topical lidocaine to the region of the marginal ear vein, the rabbits were pretreated with chlorpromazine $\mathrm{HCl}(20 \mathrm{mg}$ in $0.8 \mathrm{ml}$ of saline, i.v.) and anesthetized with sodium pentobarbital $(60 \mathrm{mg} / \mathrm{kg}, \mathrm{i} . \mathrm{v}$. $)$. The rabbits were mounted in a Kopf stereotaxic instrument and the scalp was incised. One group $(n=$ 5) received lesions of the vermis (VER). A section of skull beginning approximately $1.0 \mathrm{~mm}$ posterior to lambda and extending $3.0 \mathrm{~mm}$ on both sides of the midline and $5.0 \mathrm{~mm}$ in length was removed by drilling. The anterior lobules of the vermis were aspirated under visual guidance with the assistance of a dissecting microscope. A second group $(n=5)$ received bilateral cerebellar hemispheric aspiration lesions (HEM), made by removing bilateral sections of interparietal bone starting $3.0 \mathrm{~mm}$ lateral to the midline and extending to the external suture, and extending $4.0 \mathrm{~mm}$ posterior to lambda. These lesions were intended to remove the cortex of the anterior lobules of the cerebellar hemispheres without extending into the midline vermis or underlying dentate-interpositus nuclear complex. A third group $(n=10)$ served as an unoperated control (UNOP). Each animal's recovery was closely monitored until it regained sternal position and then was returned to its home cage.

\section{Experimental apparatus and procedure}

This experiment consisted of three phases: orienting response habituation, acquisition, and retention.

Habituation. Each rabbit was habituated to restraint by placement in a standard rabbit restrainer with adjustable head stock and backplate for five daily $30 \mathrm{~min}$ sessions. During the last two of these sessions, the rabbits were habituated to the sound-attenuating experimental chamber. This chamber was equipped with a ventilating fan that provided broadrange masking noise of $\sim 70 \mathrm{~dB}$ SPL and a speaker, and was located within a larger electrically shielded, soundproof chamber. Recording leads located within the chamber led to a Grass Instruments model 78 polygraph that recorded the heart signal as well as event markers denoting stimulus presentations. All recording and programming equipment was located outside the larger soundproof chamber.

Orienting response habituation. On the next day following the last restraint habituation session, each rabbit was fitted with ECG leads consisting of stainless steel 26 gauge wire loops positioned subcutaneously on the right front shoulder and left flank. Stainless steel 26 gauge wire loops for delivery of the UCS were inserted into the left pinna 5 $\mathrm{mm}$ medial to the marginal ear vein under local anesthesia (4.0\% topical Xylocaine). All recording leads were implanted $2 \mathrm{hr}$ prior to each training session.

Approximately 10 min after placement into the chamber, each rabbit received 20 unreinforced presentations of the tone stimulus $(5.0 \mathrm{sec}$, $1000 \mathrm{~Hz}, 90 \mathrm{~dB}$ SPL) presented on a $90 \mathrm{sec}$ variable interval (VI) intertrial schedule. These tone-alone trials were given prior to acquisition training to habituate the unconditioned HR OR that rabbits show to novel auditory stimuli. Without habituation, any bradycardiac response subsequently observed during training would be confounded with conditioned bradycardiac responses.

Acquisition training. Immediately after completion of habituation, the first acquisition phase consisting of 40 paired trials commenced. Each presentation of the CS was followed at offset by a $1.0 \mathrm{~mA}, 60 \mathrm{~Hz}$, $500 \mathrm{msec}$ pinna shock UCS; trials were presented on a $90 \mathrm{sec}$ VI schedule. The next day each animal received an additional identical 40 paired training trials, generating a total of 80 acquisition trials across $2 \mathrm{~d}$ of training.
Retention. To assess the effects of VER lesions on retention of the conditioned bradycardiac response, the UNOP group $(n=10)$ from the acquisition phase was randomly split into two equal groups: a SHAM group and a VER group. The SHAM group was anesthetized and the scalp was incised and sutured, while the VER group received aspiration lesions of the anterior vermis as described above. Five days later 20 CS-alone test (retention) trials were presented on a $90 \mathrm{sec}$ VI intertrial interval to assess retention of conditioned bradycardia.

\section{Histological procedures}

At the conclusion of behavioral testing, the lesioned rabbits were given a lethal dose of sodium pentobarbital $(120 \mathrm{mg} / \mathrm{kg}$, i.v.) and perfused intracardially with $0.9 \%$ saline followed by $10 \%$ buffered neutral formalin. The brains were removed and stored in $10 \%$ formalin for at least $48 \mathrm{hr}$. Frozen $80 \mu \mathrm{m}$ sections were taken throughout the extent of the lesions. Every fourth section was mounted and stained with thionin. The extent of the lesions was determined by camera lucida drawings.

\section{Data analysis}

HR responses were calculated by measuring the interbeat interval as indexed by successive R-waves of the cardiac cycle. HR changes during the CS (expressed in beats per minute) were computed by comparing HR during the $5.0 \mathrm{sec} C S$ from that during the $5.0 \mathrm{sec}$ pre-CS baseline period using a microcomputer system that measured the interbeat intervals with millisecond resolution. Responses following the UCS were computed by comparing HR during a $5.0 \mathrm{sec}$ period following UCS offset with the pre-CS baseline HR. The initial $1.0 \mathrm{sec}$ block after CS offset was not analyzed as shock artifact blocked amplification of the heart signal. These responses were computed by measuring successive $\mathrm{R}-\mathrm{R}$ intervals in the aforementioned periods. These intervals were read to the nearest $0.5 \mathrm{~mm}$ from the polygraph chart, which traced the heart signal on a paper speed of $25 \mathrm{~mm} / \mathrm{sec}$. To examine the topography of the HR responses during the various phases of training, the following procedures were used. The topography of the response to the tone or CS was determined by comparing the mean pre-CS HR (in beats $/ \mathrm{min}$ ) with each $1 \mathrm{sec}$ interval during the $5.0 \mathrm{sec}$ tone. The topography of the response to pinna shock was determined by comparing the mean preCS HR with each 1 sec interval for 5.0 sec commencing after the initial $1.0 \mathrm{sec}$ shock artifact period described above. Repeated-measures analyses of variance (ANOVA) were performed on the beats per minute values. Post hoc Neuman-Keuls pairwise comparisons between means were performed where appropriate.

\section{Results}

\section{Recovery}

The typical postoperative recovery of the cerebellar-lesioned rabbits was rapid and unremarkable. There were no persistant postural or obvious motor impairments, other than several VER animals showing a mild ataxia that dissipated in 1 or $2 \mathrm{~d}$ postsurgery.

\section{Histological analysis}

Serial reconstructions of representative VER and HEM lesions are presented in Figure 1. Lesions of the vermis were restricted to the anterior midline of the cerebellum primarily involving the culmen (lobules IV, V, and VI according to the terminology of Brodal, 1940) with some damage to the more anteriorly located central lobule (lobule III). The underlying fastigial nuclei were not invaded or damaged. Lesions of the cercbellar hemispheres were bilaterally symmetrical, with damage confined to the lateral ansiform and paramedian lobules (Crus 1). There was no damage to either the interpositus or dentate nucleus. There was no extracerebellar damage in either group. These two lesion groups are essentially complementary in that there was no overlap of involved tissue between the VER and HEM groups.

\section{Heart rate}

Baseline heart rate. All three groups showed similar and consistent patterns of baseline HR during acquisition training. The 


\section{VERMIS}

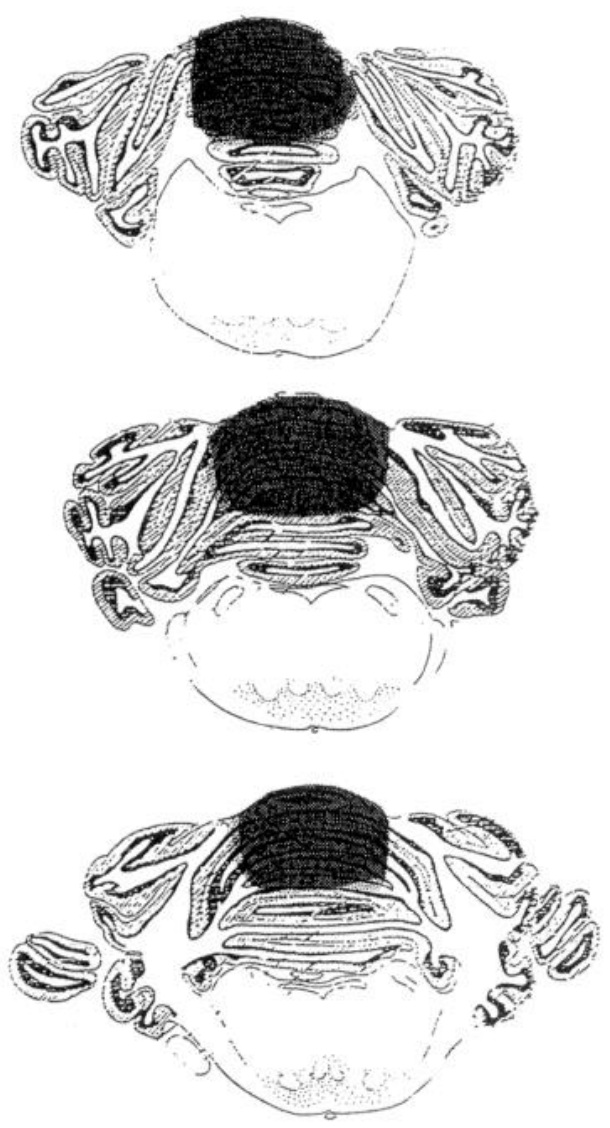

\section{HEMISPHERE}

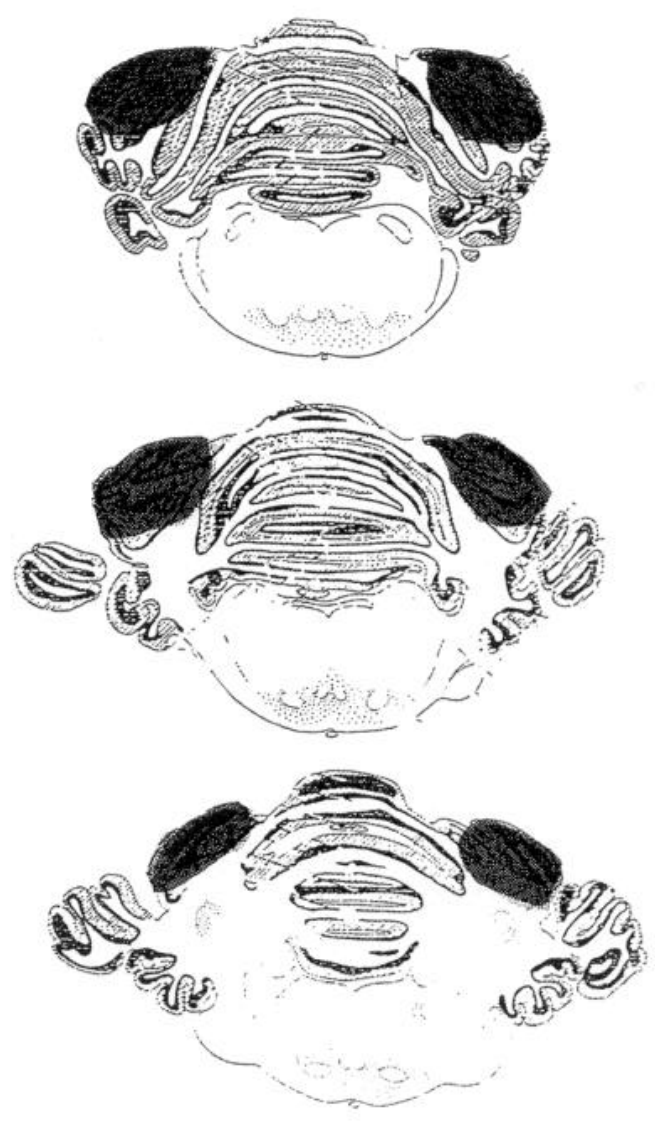

Figure 1. Serial reconstructions showing representative examples of the extent of damage (darkly shaded area) following cerebellar vermis and bilateral cerebellar hemispheric lesions. Note that these two lesion groups are essentially complementary with no overlap of involved cerebellar tissue between the two groups. mean baseline HRs for the UNOP, VER, and HEM groups were $232.6(\mathrm{SD}=22.3), 230(\mathrm{SD}=25.6)$, and $221.9(\mathrm{SD}=17.7)$ beats $/ \mathrm{min}$, respectively. There were no significant group differences in baseline HR assessed in five-trial blocks across the 2 $\mathrm{d}$ of acquisition training $[F(2,17)=0.78, p>0.27]$, suggesting no effect of these lesions on resting or baseline HR.

$H R O R$ and habituation. The initial HR response to the tone in all the animals consisted of bradycardia that habituated across repeated tone presentations. The magnitude and subsequent habituation of this OR were similar in all groups. Figure $2 A$ presents each group's mean HR response to the tone during this phase of training. The initial response was a deceleration that habituated over trial blocks $[F(3,51)=45.17, p<0.01]$. The main effect of groups was not significant $[F(2,17)=0.49]$, nor was the groups $\times$ blocks interaction $[F(6,51)=1.22, p>0.32]$. No group differences emerged when the topography of the HR OR during the $5 \mathrm{sec}$ tone was examined $(F$ values $<1)$. These results indicate that VER or HEM lesions did not disrupt the initial magnitude, topography, or habituation characteristics of the bradycardiac HR OR.

Acquisition of conditioned bradycardia. Figure $2 B$ presents the data for each group across the $2 \mathrm{~d}$ of acquisition training. Inspection of Figure $2 B$ shows that both the UNOP and HEM groups developed robust bradycardiac responses during the fear conditioning procedure. In contrast, lesions of the ACV severely impaired the acquisition of conditioned bradycardia. This pattern of responding resulted in a significant main effect of groups $[F(2,17)=8.47, p<0.01]$, trial blocks $[F(7,119)=7.59, p<$
$0.01]$, and a groups $\times$ trial blocks interaction $[F(14,119)=4.32$, $p<0.01]$, on the first day of acquisition training. This pattern continued on the second day of training as reflected by a significant main effect for groups $[F(2,17)=11.23, p<0.01]$. Separate Newman-Keuls comparisons showed that the VER group demonstrated significantly less bradycardia during the CS compared to either the UNOP or HEM group on both days (all $p$ values $<0.05)$.

The topography of the HR response during the CS was also examined. Figure 3 presents the second-by-second topography of the HR response of each group collapsed across trials and days. The response of both the UNOP and HEM group was markedly different than that of the VER group. An overall ANOVA found a significant groups $\times$ CS periods interaction $[F(8,136)$ $=3.79, p<0.05]$. This interaction reflected the development of greater bradycardia during the latter seconds of the CS for the UNOP and HEM groups, while the VER group demonstrated minimal bradycardiac responses during the CS. This finding illustrates that the VER group, in addition to showing minimal bradycardia during the CS, also showed little evidence of a within-CS pattern that resembled that of the two other groups.

Retention of conditioned bradycardia. To assess the contribution of the anterior vermis to the retention of conditioned bradycardia, the UNOP control group from the acquisition phase was randomly divided into two groups. Half received VER lesions; half served as surgical controls (SHAM operations). Figure $2 C$ shows the effects of lesions of the anterior vermis performed after the acquisition of conditioned bradycardia 


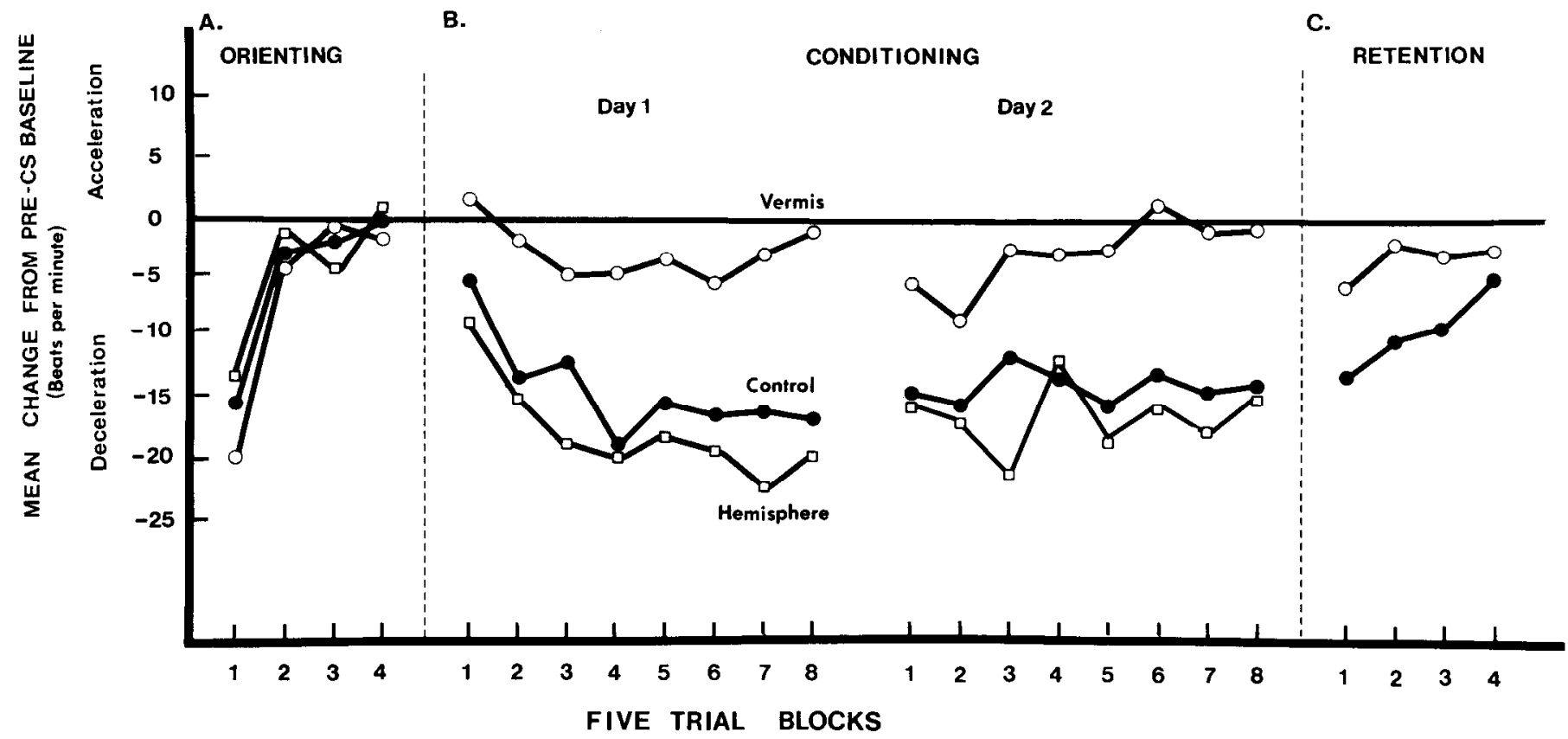

Figure 2. The effects of cerebellar VER and HEM lesions on the HR OR, acquisition of the HR CR, and retention of the CR. $A$, HR OR. Shown is the mean HR change in beats/min during the $5.0 \mathrm{sec}$ tone period compared to the $5.0 \mathrm{sec}$ baseline period in blocks of five trials. Deceleration is indicated by negative numbers along the vertical axis. Note that the initial response to the tone was bradycardiac that habituated across trials. Cerebellar lesions had no effect on this response. $B$. Acquisition of conditioned bradycardia. UNOP (Control) and HEM rabbits acquired conditioned bradycardia while the VER group did not across the $2 \mathrm{~d}$ of acquisition training. $C$, Retention of conditioned bradycardia. The control group from the acquisition phase was split into two groups, one group received VER lesions the other SHAM lesions. After a $5 \mathrm{~d}$ recovery period, the retention of conditioned bradycardia was assessed under extinction conditions. Initially, the SHAM animals demonstrated bradycardia to the CS that subsequently extinguished. The VER group did not show bradycardia, suggesting that the lesion eliminated evidence of prior conditioning.

compared to SHAM-operated controls. The SHAM group showed an initial bradycardia during the CS-alone retention trials that subsequently extinguished. These data suggest that intact rabbits retain conditioned bradycardia across this time interval and that surgery alone did not disrupt conditioned bradycardia established during the acquisition phase of training. In contrast, lesions of the vermis severely reduced evidence of prior conditioned responding and attenuated the magnitude of conditioned bradycardia to a level observed in rabbits with lesions performed prior to acquisition training (compare VER group, Fig. $2 B$, with VER group, Fig. $2 C$ ). This pattern of responding generated significant main effects for groups $[F(1,8)=9.86, p$ $<0.05]$, trial blocks $[F(3,24)=6.97, p<0.05]$, and a groups $\times$ trial blocks interaction $[F(3,24)=3.71, p<0.05]$, for the retention phase of training.

Unconditioned IIR response to pinna UCS. The HR UCR consisted of a monophasic tachycardiac response. The mean tachycardiac response of each group in beats/min was UNOP $9.33(\mathrm{SD}=16.45), 13.78(\mathrm{SD}=13.24)$; HEM $13.49(\mathrm{SD}=$ 12.79), 14.87 (SD = 14.22); and VER 13.88 (SD = 19.34), 16.23 $(\mathrm{SD}=18.20)$, for days 1 and 2 of acquisition training, respectively. Importantly, neither the magnitude of the UCR nor the pattern over acquisition trials was affected by these cerebellar lesions. Analysis of blocks of five acquisition trials across both days of training resulted in no significant main effects for groups $[F(2,17)=0.42, p>0.65]$, trial blocks $[F(15,255)=1.294, p$ $>0.20]$, or groups $\times$ trials blocks interaction $[F(30,255)=0.47$, $p>0.80]$. Also, examination of the topographical characteristics of the HR response following the pinna shock revealed a similar pattern of responding between the three groups. As shown in Figure 4 , the second-by-second pattern consisted of a mono- phasic tachycardia peaking at the second measurement interval. An overall ANOVA of these data revealed a significant main effect for measurement periods $[F(4,316)=3.87, p<0.05]$, reflecting the change across measurement periods; however, no main effects or interactions involving groups were significant (all $p$ values $>0.23$ ). Overall, these results indicated that VER lesions, which disrupted conditioned HR responses, did not disrupt unconditioned $\mathrm{HR}$ responses to the pinna shock UCS used in conditioning.

\section{Discussion}

This study demonstrated that lesions of the ACV severely disrupted the acquisition and retention of simple Pavlovian fearconditioned bradycardiac responses in the rabbit. The aversive HR conditioning paradigm is a useful model system for the analysis of the neural substrates of Pavlovian fear conditioning because it allows for the assessment of unconditioned HR responsiveness after brain lesions. An advantage of studying a response system like conditioned bradycardia is that it is relatively simple to detect manipulation-induced sensory or motor impairments that could interfere with the detection of the stimuli used in training or interruption of the efferent pathway for the CR (e.g., Schneiderman et al., 1986; Pascoe et al., 1991). The unconditioned HR responses of rabbits with ACV lesions were not disrupted. Baseline or resting IIR was not altered, nor was the magnitude, topography, or habituation characteristics of the HR OR to the unreinforced tone presentations. These data suggest that the observed conditioning deficit following ACV lesions was probably not secondary to brain lesion-induced auditory impairments or to a disruption of the rabbit's ability to demonstrate stimulus-evoked bradycardia. The bra- 

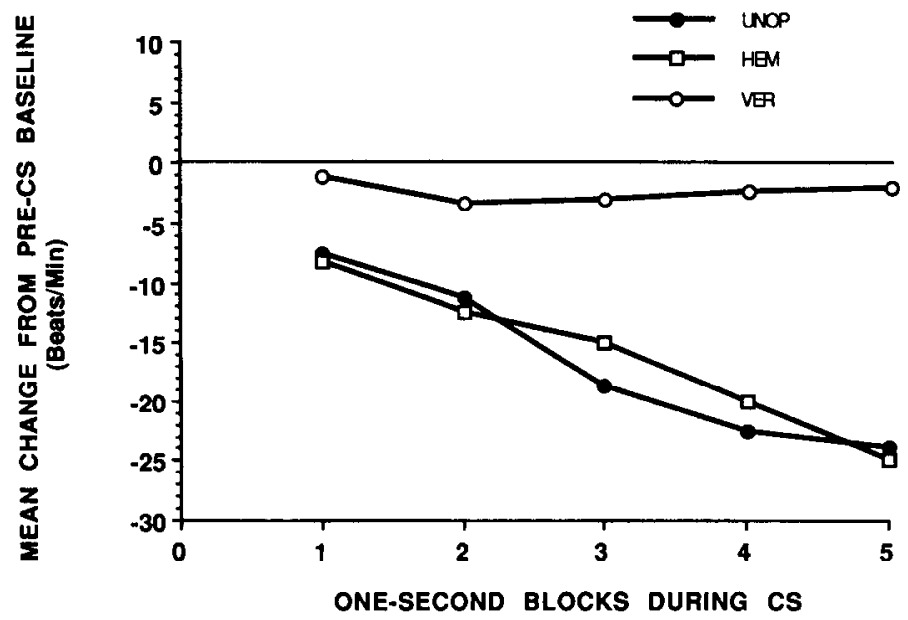

Figure 3. Topography of the conditioned HR response. Shown is the mean change in HR (beats/min) during each $1.0 \mathrm{sec}$ period of the 5.0 sec CS collapsed across days and trials for the UNOP, HEM, and VER groups. Note the difference in the topography of the response of the VER group compared to the UNOP and HEM groups. The HEM and UNOP groups demonstrated progressively greater bradycardia through the CS period, while the VER group showed minimal bradycardia during all periods of the CS.

dycardiac responses to the novel auditory stimulus indicated that the animal heard the stimulus and could unconditionally decelerate the heart. ACV lesions also did not disrupt the magnitude or topographical features of the unconditioned tachycardiac responses to the pinna shock UCS. The results indicating intact UCRs following vermis lesions in rabbits are consistent with previous data in rats (Peters et al., 1973; Supple and Leaton, $1990 \mathrm{a}, \mathrm{b})$. Overall, these results suggest that the ACV lesions did not produce nonspecific primary sensory or cardiomotor impairments that could interfere with the development of the conditioned bradycardiac response. Rabbits with ACV lesions can hear the tone CS, respond similarly to the shock UCS, and unconditionally decelerate the heart. Given the selective elimination of conditioned but not unconditioned HR responses, these lesion results are consistent with the hypothesis that the $\mathrm{ACV}$ is a component of a neural circuit importantly involved in classically conditioned bradycardiac responses in the rabbit.

Rabbits with ACV lesions showed minimal CRs during both acquisition and retention. This minimal response consisted of a 3-4 beats/min bradycardia, which was in contrast to the typical 18-20 beats/min bradycardia shown by the UNOP and HEM groups. This minimal bradycardia in the VER group was very similar in magnitude to that shown by explicitly unpaired or pseudoconditioned control groups from previous studies conducted in this laboratory and others (e.g., Gallagher et al., 1981; Jarrell et al., 1986). In addition to the greatly reduced overall magnitude of conditioned bradycardia was the relative lack of topographical features characteristic of conditioned responding for this autonomic response in the VER group. In summary, ACV lesions severely attenuated the overall magnitude of the $\mathrm{CR}$ and blocked the appearance of the topographical features of the CR that were present in the control animals.

The demonstrated role of the cerebellum in motor coordination and regulation raises the possibility that a subtle motor impairment could somehow be responsible for the observed autonomic conditioning deficit. The lesion results from the surgical control group (HEM) provided evidence against this pos-

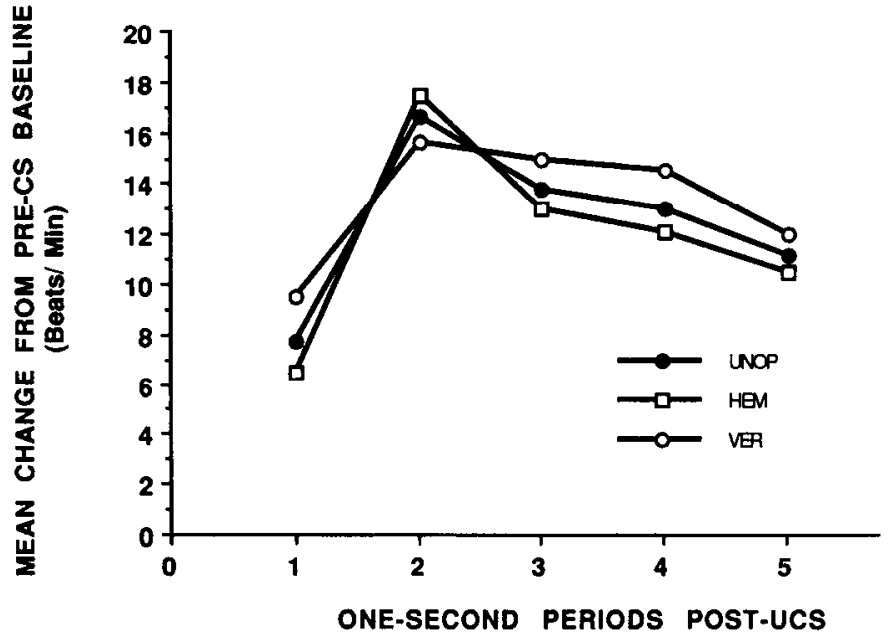

Figure 4. Topography of the HR response following application of the pinna shock UCS during acquisition training. Shown is the mean change in HR (beats $/ \mathrm{min}$ ) during each $1.0 \mathrm{sec}$ period following the offset of the UCS collapsed across days and trials. Note the monophasic acceleration in HR after the UCS, and the similarity in response profiles among the three groups.

sibility as HEM lesions did not disrupt conditioned bradycardia despite any subtle, undetectable motor disruptions that may have resulted from these cerebellar lesions. Therefore, these findings suggest that cerebellar damage per se does not disrupt conditioned bradycardia and generate support regarding the anatomical specificity for the effect consistent with the hypothesis that the ACV is importantly involved in this lcarncd autonomic response.

The present results implicating the cerebellar vermis in HR conditioning are not without precedent, and are consistent with those obtained with rats (Supple, 1986; Supple and Leaton, 1990a,b). Large lesions of the vermis blocked the acquisition of simple and discriminatively conditioned $H R$ responses without disrupting unconditioned HR responses in rats. Furthermore, bilateral cerebellar hemispheric lesions also did not affect HR CRs, which is consistent with the data in the rabbit. The present results extend those previous data on the following points: (1) replicating the effect of vermis lesions on HR CRs in the rabbita species with a wealth of accumulated data regarding the neural substrates of classical conditioning (e.g., Schneiderman et al., 1986), (2) providing partial anatomical localization for the behavioral effect to the $\mathrm{ACV}$, and (3) demonstrating that the ACV is importantly involved in retention of the $\mathrm{HR} C R$ as well as acquisition. Regarding the last point, it is notable that ACV lesions blocked the retention of the bradycardiac CR. Disruption of a previously acquired response following brain damage, coupled with the selective abolition of CRs but not UCRs in acquisition training, may be convincing behavioral evidence that the ACV is critically important for this CR. Furthermore, singleunit electrophysiological recordings of Purkinje cell activity during retention trials allowed us to identify several distinct subpopulations of ACV Purkinje cells that show short-latency differential responses to tone stimuli trained as $\mathrm{CS}^{+}$and $\mathrm{CS}^{-}$ (Supple and Kapp, 1988). Moreover, a group of Purkinje cells showed significant correlations between discharges during the $\mathrm{CS}^{+}$and the magnitude of the concomitant conditioned bradycardiac response (Supple and Kapp, 1988), suggesting possible ACV mediation of the amplitude-time course of this autonomic 
$\mathrm{CR}$. These electrophysiological results, along with the lesion data from the present study, provide compelling evidence in support of the hypothesis that the ACV has an important functional contribution to the acquisition and expression of the classically conditioned bradycardiac response in the rabbit.

A related question concerns the location of the $\mathrm{ACV}$ in a $\mathrm{CR}$ circuit for conditioned bradycardia; is it located on the sensory or motor side of a CR circuit, efferent to a site of essential plasticity or a candidate site of stimulus convergence-a possible site of primary neural plasticity? Although answers to these questions cannot be derived from lesion experiments alone, there are findings from the present study that may be useful when considering these issues. In light of the finding of intact UCRs following ACV lesions in the present study, it is probable that the ACV is not part of a primary or fundamental response system for the auditory CS or pinna shock UCS. However, there are several reasons to consider the ACV as a site for stimulus convergence in this response system. First, the ACV receives abundant sensory input (e.g., Fadiga and Pupilli, 1964; Aitkin and Boyd, 1975; Ito, 1984) and has extensive output to both brainstem and forebrain regions involved in cardiovascular regulation (e.g., Ito, 1984; Supple and Kapp, 1993). Second, the cerebellar cortex has well-defined and orderly sensory input and motor output pathways. The two major types of input to the cortex via mossy and climbing fibers may provide the anatomical substrate for stimulus convergence necessary for some forms of associative learning. This anatomical parcellation has suggested to others that the cerebellum may be a promising site for sensorimotor learning (e.g., Marr, 1969; Gilbert, 1975), and has recently been extensively studied in the context of the contributions of the lateral cerebellar hemisphere to aversively conditioned nictitating membrane responses in the rabbit (Thompson, 1986; Steinmetz and Sengelaub, 1992). Finally, we have obtained electrophysiological evidence of convergence onto single ACV Purkinje cells of the two exact stimulus modalities used to condition HR in our preparation. Single-unit recordings of Purkinje cells showed short-latency UCRs to the prospective CSs prior to training, and these responses subsequently habituated over repeated presentations of the tones. Also, some Purkinje cells demonstrated short-latency UCRs to brief presentations of pinna shock stimulation prior to training. Studies in progress are examining the relationships between these neuronal responses and the conditioned bradycardiac response in the rabbit.

The present results, and a number of previous observations, are consistent with the hypothesis that the ACV is part of a more extensive neuroanatomical system involved in the acquisition and expression of conditioned bradycardia and possibly other fear-conditioned autonomic responses as well (discussed at length in Supple and Leaton, 1990b). This suggestion is consistent with the growing appreciation of the neuroanatomical overlap the ACV shares with other forebrain regions importantly involved in conditioned bradycardia, most notably the amygdaloid central nucleus $(\Lambda C E)$. Converging lines of evidence implicate the ACE in conditioned bradycardia in the rabbit. Lesions or pharmacological manipulations of the ACE severely attenuated the acquisition of conditioned bradycardia without disrupting HR UCRs (Kapp et al., 1982). Recordings from ACE neurons revealed the presence of associative responses to a fearconditioned CS (Applegate et al., 1982; Pascoe and Kapp, 1985). The ACE has extensive anatomical connections with cardioregulatory nuclei, thereby suggesting pathways through which al- tered ACE neuronal activity could affect HR (Schwaber et al., 1980). In addition to involvement in this autonomic CR, the ACE also importantly contributes to some somatomotor responses that are acquired as a result of fear conditioning procedures. Lesions of the ACE severely impair conditioned freezing (Iwata et al., 1986) and potentiated acoustic startle responses (Hitchcock and Davis, 1986), thus suggesting a wider role of the ACE in the generation of a conditioned central state of fear.

It is interesting that experimental manipulations of two seemingly disparate neuroanatomical systems, the ACV and ACE, result in strikingly similar effects on conditioned bradycardia in the rabbit. These similarities suggest the possibility that these two rcgions are components of a common, more extensive neuroanatomical system involved in this response. Recent anatomical observations lend support to this hypothesis. Both the ACE and $\mathrm{ACV}$ receive afferents from common sources, the pontine parabrachial nucleus (Dietrichs, 1985) and the lateral hypothalamus (Dietrichs and Haines, 1986, 1989), both of which have been implicated in cardiovascular function. Furthermore, we have recently demonstrated that single-pulse electrical microstimulation of either the lateral parabrachial nucleus or the lateral hypothalamus modulates Purkinje cell discharges in the rabbit ACV (Supple and Kapp, 1993; Supple, in press), suggesting the possibility that these structures may serve as an interface between the ACV and other brain regions importantly involved in conditioned bradycardia. Further experiments will be required to examine the nature of the information relayed by these afferents into the ACV and their functional relevance to conditioned bradycardia, and the specific contributions of the ACV within the context of this larger neuroanatomical system to aversively conditioned bradycardiac responses.

\section{References}

Aitkin LM, Boyd J (1975) Responses of single units in cerebellar vermis of the cat to monaural and binaural stimuli. J Neurophysiol 38:418-429.

Albert TJ, Dempesy CW, Sorenson CA (1985) Anterior cerebellar vermal stimulation: effect on behavior and basal forebrain neurochemistry in rat. Biol Psychiatry 20:1267-1276.

Applegate CD, Frysinger RC, Kapp BS, Gallagher M (1982) Multiple unit activity recorded from the amygdala central nucleus during Pavlovian heart rate conditioning in the rabbit. Brain Res 238:457-462.

Asdourian D, Frerichs K (1970) Some effects of cerebellar stimulation. Psychonom Sci 18:261-262.

Berman AJ, Berman D, Prescott JW (1974) The effect of cerebellar lesions on emotional behavior in the rhesus monkey. In: The cerebellum, epilepsy, and behavior (Cooper IS, Riklan M, Snider RS, eds), pp 277-284. New York: Plenum.

Berntson GG, Torello MW (1982) The paleocerebellum and the integration of behavioral function. Physiol Psychol 10:2-12.

Brodal A (1940) The cerebellum of the rabbit. A topographical atlas of the folia as revealed in transverse sections. J Comp Neurol 72:6381 .

Chida K, Iadecola C, Underwood MD, Reis DJ (1986) A novel vasodepressor response elicited from the rat cerebellar fastigial nucleus: the fastigial depressor response. Brain Res 370:378-382.

Dietrichs E (1985) Divergent axon collaterals to cerebellum and amygdala from neurons in the parabrachial nucleus, the nucleus locus coeruleus and some adjacent nuclei: a fluorescent double labeling study using rhodamine labeled latex microspheres and fast blue as retrograde tracers. Anat Embryol (Berl) 172:375-382.

Dietrichs E, Haines DE (1986) Do the same hypothalamic neurons project to both amygdala and cerebellum? Brain Res 364:241-248.

Dietrichs E, Haines DE (1989) Interconnections between hypothalamus and cerebellum. Anat Embryol (Berl) 179:207-220.

Dow RS, Moruzzi G (1958) The physiology and pathology of the cerebellum. Minneapolis: University of Minnesota. 
Fadiga E, Pupilli GC (1964) Teleceptive components of cerebellar function. Physiol Rev 44:432-486.

Gallagher M, Kapp BS, McNall CL, Pascoe JP (1981) Opiate effects in the amygdala central nucleus on heart rate conditioning in rabbits. Pharmacol Biochem Behav 14:497-505.

Gilbert PFC (1975) How the cerebellum could memorise movements. Nature 254:688-689.

Hitchcock J, Davis M (1986) Lesions of the amygdala, but not of the cerebellum or red nucleus, block conditioned fear as measured with the potentiated startle paradigm. Behav Neurosci 100:11-22.

Hockman CH, Livingston KE, Talesnik J (1970) Cerebellar modulation of reflex vagal bradycardia. Brain Res 23:101-104.

Hoffer BJ, Mitra J, Snider RS (1972) Cerebellar influences on the cardiovascular system. In: Limbic system mechanisms and autonomic function (Hockman CH, ed). Springfield, IL: Thomas.

Ito $M$ (1984) The cerebellum and neural control. New York: Raven.

Iwata J, LeDoux JE, Meeley MP, Arneric S, Reis DJ (1986) Intrinsic neurons in the amygdaloid field projected to by the medial geniculate body mediate emotional responses conditioned to acoustic stimuli. Brain Res 383:195-214.

Jarrell TW, McCabe PM, Teich A, Gentile CG, VanDercar DH, Schneiderman N (1986) Lateral subthalamic area as mediator of classically conditioned bradycardia in rabbits. Behav Neurosci 100:3-10.

Kapp BS, Frysinger RC, Gallagher M, Haselton JR (1979) Amygdala central nucleus lesions: effects on heart rate conditioning in rabbits. Physiol Behav 23:1 109-1117.

Kapp BS, Gallagher M, Applegate CD, Frysinger RC (1982) The amygdala central nucleus: contributions to conditioned cardiovascular responding during aversive Pavlovian conditioning in the rabbit. In: Conditioning: representation of involved neural functions (Woody CD, ed). New York: Plenum.

Lavond DG, Lincoln JS, McCormick DA, Thompson RF (1984) Effects of bilateral lesions of the dentate and interpositus cerebellar nuclei on conditioning of heart rate and nictitating membrane/eyelid responses in the rabbit. Brain Res 305:323-330.

Marr D (1969) A theory of cerebellar cortex. J Physiol (Lond) 202: 437-470.

Martner M (1975) Cerebellar influences on autonomic mechanisms: an experimental study in the cat with special reference to the fastigial nucleus. Acta Physiol Scand [Suppl] 425:1-42.

McCormick DA, Thompson RF (1984) Cerebellum: essential involvement in the classically conditioned eyelid response. Science 223:296299.

Miura M, Reis DJ (1969) Cerebellum: a pressor response elicited from the fastigial nucleus and its efferent pathway in the brainstem. Brain Res 13:595-599.

Moruzzi G (1940) Paleocerebellar inhibition of vasomotor and respiratory carotid sinus reflexes. J Neurophysiol 3:20-32.

Nisimaru N, Watanabe Y (1985) Inhibitory effects of cerebellar nodulus stimulation on sympathetic nerve activity and systemic blood pressure in rabbit. Nippon Seirigaku Zasshi 47:447.

Pascoe JP, Kapp BS (1985) Electrophysiological characteristics of amygdaloid central nucleus neurons during Pavlovian fear conditioning in the rabbit. Behav Brain Res 16:117-133.

Pascoe JP, Supple WF Jr, Kapp BS (1991) Learning and memory: vertebrate models. In: Learning and memory: a biological view, 2d ed (Martinez JL, Kesner RP, eds), pp 359-400. New York: Academic.

Peters M, Monjan AA (1971) Behavior after cerebellar lesions in cats and monkeys. Physiol Behav 6:205-206.

Peters M, Bleek C, Monjan AA (1973) Reaction to electric shock after cerebellar lesions in the rat. Physiol Behav 10:429-433.

Reis DJ, Doba N, Nathan MA (1973) Predatory attack, grooming, and consummatory behaviors evoked by electrical stimulation of cat cerebellar nuclei. Science 182:845-847.

Schneiderman N, McCabe PM, Haselton JR, Ellenberger HH, Jarrell TW, Gentile CG (1986) Neurobiological bases of conditioned bradycardia in the rabbit. In: Classical conditioning III (Gormezano I, Prokasy WF, Thompson RF, eds), pp 37-63. Hillsdale, NJ: Erlbaum.

Schwaber JS, Kapp BS, Higgins GA (1980) The origin and extent of direct amygdala projections to the region of the dorsal motor nucleus of the vagus and nucleus of the solitary tract. Neurosci Lett 20:1520.

Snider RS (1972) Some cerebellar influences on autonomic function. In: Limbic system mechanisms and autonomic function (Hockman $\mathrm{CH}$, ed). Springfield, IL: Thomas.

Steinmetz JE, Sengelaub DR (1992) Possible conditioned stimulus pathway for classical eyelid conditioning in rabbits. I. Anatomical evidence for direct projections from the pontine nuclei to the cerebellar interpositus nucleus. Behav Neural Biol 57:103-115.

Supple WF Jr (1986) The effects of lesions of the cerebellar vermis and cerebellar hemispheres on conditioned and unconditioned heartrate responses in restrained and unrestrained rats. $\mathrm{PhD}$ thesis, Dartmouth College.

Supple WF Jr (1993) Hypothalamic modulation of Purkinje cell activity in the anterior cellebellar vermis. Neuroreport 4:in press.

Supple WF Jr, Kapp BS (1988) Purkinje cell responses in the cerebellar vermis during Pavlovian fear conditioning in the rabbit. Soc Neurosci Abstr 14:395.

Supple WF Jr, Kapp BS (1993) Anatomical and electrophysiological relationships between the anterior cercbcllar vermis and the pontine parabrachial nucleus in the rabbit. Brain Res Bull, in press.

Supple WF Jr, Leaton RN (1990a) Cerebellar vermis: essential for classically conditioned bradycardia in rats. Brain Res 509:17-23.

Supple WF Jr, Leaton RN (1990b) Lesions of the cerebellar vermis and cerebellar hemispheres: effects on heart-rate conditioning in rats. Behav Neurosci 104:934-947.

Supple WF Jr, Fanselow MS, Leaton RN (1987) Effects of cerebellar vermal lesions on species-specific fear responses, neophobia, and tasteaversion learning in rats. Physiol Behav 39:579-586.

Supple WF Jr, Cranney J, Leaton RN (1988) Effects of lesions of the cerebellar vermis on VMH-lesioned induced hyperdefensiveness, spontaneous mouse killing, and freezing in rats. Physiol Behav 42: $145-153$.

Supplc WF Jr, Archer L, Kapp BS (1989) Lesions of the cerebellar vermis severely disrupt classically conditioned bradycardia in rabbits. Soc Neurosci Abstr 15:978.

Thompson RF (1986) The neurobiology of learning and memory. Science 233:941-947.

Yeo CH, Hardiman MJ, Glickstein M (1985) Classical conditioning of the nictitating membrane response in the rabbit. II. Lesions of the cerebellar cortex. Exp Brain Res 60:99-113. 